\title{
Etude de la compressibilité et de la microstructure d'un sédiment de dragage traité au liant hydraulique.
}

\author{
Boubaker Rekik, Mohamed Boutouil
}

\section{Ecole Supérieure d'Ingénieurs des Travaux de la Construction de Caen - 1, rue Pierre et Marie Curie, 14610, Epron.}

\begin{abstract}
RESUME. La solidification à base de ciment est une technique largement utilisée pour le traitement des sédiments de dragage. Elle permet la valorisation des matériaux traités notamment en applications géotechniques. Dans cette étude, des essais de compactage et de compressibilité ont été effectués sur des sédiments non traités et traités avec 10\% de ciment. Les résultats montrent que le traitement entraîne une amélioration du comportement au compactage avec une augmentation de l'indice portant immédiat qui atteint 11. Le suivi des paramètres de compressibilité dans le temps montre le passage du comportement d'un sédiment très compressible et sans pression de consolidation à celui d'un matériau traité avec une pression de préconsolidation qui augmente dans le temps pour atteindre $250 \mathrm{kPa}$. Sous l'effet de la cimentation, le temps de consolidation et la consolidation secondaire sont également réduits. Les analyses au microscope électronique à balayage et par diffractométrie aux rayons $X$ ont révélé des transformations microstructurales pouvant être corrélées aux modifications du comportement géotechnique.
\end{abstract}

MOTS-CLÉS : sédiment, compressibilité, solidification.

ABSTRACT. Cement-based solidification is one of the most used treatment techniques for dredged sediment. It allows geotechnical applications of the treated sediment. In this paper, compaction and compressibility tests were carried out to investigate a part of the geotechnical behavior of the treated and non-treated sediment. Compaction and compressibility parameters were determined for different curing periods of sediment treated with $10 \%$ cement. compaction test results show an enhancement in the compaction characteristics. Results indicate that the sediment had no preconsolidation pressure. After treatment, samples showed a preconsolidation pressure which increases with curing time and achieves $250 \mathrm{kPa}$. Overall and under cementation effect, treated sediment was found to have lower consolidation time and secondary consolidation. Furthermore, compaction test results show an enhancement in the compaction characteristics. Compared with non-treated sediment, microstructure (SEM and XRD) of treated sediment was found denser and could explain a part of the geotechnical behavior.

KEYWORDS : sediment, compressibility, solidification.

\section{INTRODUCTION}

Les opérations de dragage des installations portuaires concernent le plus souvent des matériaux ayant une fraction prédominante très fine. A l'extraction, les sédiments sont très humides et ne montrent aucune résistance mécanique. Le traitement des sédiments par les liants hydrauliques (Colin, 2003 ; Dermatas et al., 2003 ; Bennert et al., 2000 ; Boutouil, 1998) essentiellement la chaux et/ou le ciment, permet d'améliorer leurs caractéristiques mécaniques et géotechniques. Ces améliorations se traduisent par une augmentation (i) de la résistance à la compression et au cisaillement (Bennert et al., 2000 ; Boutouil, 1998 ), (ii) des indices portants immédiats ou indices CBR (Colin, 2003 ; Bennert et al., 2000). Une réduction de la plasticité par floculation par apport du calcium puis cimentation est

DOI: 10.5150/jngcgc.2006.061-R (disponible en ligne - http://www.paralia.fr - available online)


aussi observée et est attribuée à la formation d'hydrates, essentiellement les silicates de calcium hydratés (C-S-H) et des aluminates de calcium hydratés (C-A-H), résultants des réactions pouzzolaniques des silicates et des aluminates avec la chaux ou de l'hydratation du ciment lorsque celui-ci est utilisé. Si la chaux est souvent privilégiée dans le traitement des sols fins du fait des coûts relativement faibles, le traitement au ciment présente l'avantage d'être d'un effet rapide et de conduire à de meilleures résistances mécaniques (Tao- Wei Feng et al. 2001).

Dans le cas des sédiments de dragage, le traitement par des liants hydrauliques ne peut être effectué qu'après réduction des teneurs en eau par un processus de déshydratation. Cette opération reste difficile et coûteuse vu les volumes importants dragués. Néanmoins, le traitement des sédiments immédiatement après extraction est une solution qui a montré son efficacité, par exemple pour des sédiments des ports de New York et New Jersey (Dermatas, 2003 ; Bennert et al., 2001). La valorisation des sédiments fins dans la réalisation des remblais nécessite une amélioration de la résistance mécanique mesurée à travers la portance (GTS, 2000). Or les tassements différentiels sont également des problèmes rencontrés dans le cas des sols fins. Différentes techniques sont utilisées pour améliorer le comportement en compressibilité de ces sols. Le traitement par des liants hydrauliques permet outre l'augmentation de la résistance mécanique, l'amélioration du comportement en compressibilité des sols par augmentation des pressions de préconsolidation et réduction de la consolidation secondaire (Tao-Wei Feng et al. 2001 ; Tremblay et al., 2001).

Dans le cadre de travaux de recherche sur les possibilités de valorisation des sédiments de dragage dans la réalisation de remblai, l'étude présentée ici s'intéresse aux propriétés géotechniques de sédiment traité au ciment afin de d'évaluer le comportement au compactage et à la compressibilité. Des analyses de la microstructure du matériau traité, par diffractométrie aux rayons $\mathrm{X}$ et microscopie électronique à balayage, complètent l'étude.

\section{MATERIAUX}

Le sédiment utilisé dans cette étude provient du port de Ouistreham (Calvados). Sa granulométrie a été étudiée à l'aide d'un granulomètre laser. Il en ressort que la fraction inférieure à $63 \mu \mathrm{m}$ (argile et limon) est dominante et représente près de $86 \%$. La fraction argileuse $(0-2 \mu \mathrm{m})$ constitue $10 \%$, le limon $76 \%$ et le sable $14 \%$. Le calcul des coefficients d'uniformité, $\mathrm{C}_{\mathrm{u}}=3,3(<4)$ et de courbure, $\mathrm{C}_{\mathrm{c}}=0,7(<1)$ montre une granulométrie serrée et un sédiment mal gradué. D'après le Tableau 1 qui présente d'autres paramètres de caractérisation du sédiment, on constate tout d'abord une teneur en eau très élevée, mais qui demeure caractéristique d'un sédiment de dragage portuaire. Le sédiment brut peut également être caractérisé par une plasticité très importante. C'est une conséquence probable de sa finesse et d'une teneur en matières organiques élevée (9\%). En effet, les limites de liquidité et de plasticité augmentent avec l'augmentation de la quantité de matières organiques (Rashid et Brown, 1975). Keller (1982) a montré qu'une quantité de 3 à $4 \%$ de matières organiques était nécessaire pour modifier les caractéristiques du sol, modifications qui dépendent de la nature des matières organiques (Bennett et al., 1985 dans Tremblay, 2001).

Le sédiment brut (avant traitement) a également fait l'objet d'analyses microstructurales, par diffractométrie aux rayons $\mathrm{X}$ (DRX) et par microscopie électronique à balayage (MEB) et d'une étude de son comportement au compactage et en compressibilité. Les résultats de ces analyses sur le 
sédiment avant traitement seront présentés ultérieurement dans la partie relative à la discussion sur les résultats concernant le sédiment traité.

\begin{tabular}{|l|l|}
\hline Teneur en eau moyenne $w(\%)$ & 127 \\
\hline Teneur moyenne en matières organiques $(\%)$ & 9 \\
\hline Limite de liquidité $w_{l}(\%)$ & 104 \\
\hline Limite de plasticité $w_{p}(\%)$ & 38 \\
\hline Indice de plasticité $\mathrm{I}_{\mathrm{p}}$ & 66 \\
\hline Densité des grains solides & 2,40 \\
\hline $\mathrm{pH}$ & 7,6 \\
\hline
\end{tabular}

Tableau 1 : Caractéristiques du sédiment utilisé.

\section{METHODES}

Afin d'étudier la possibilité de valorisation des sédiments fins de dragage dans la réalisation des remblais, des essais de compactage et de compressibilité ont été effectués sur un sédiment non traité et, selon des études effectuées par, Dermatas (2003), Bennert et al. (2001) et Boutouil (1998), traité avec $10 \%$ de ciment CEM II/B-M (LL-S) de classe 32.5 (dosage exprimé par rapport à la masse humide du sédiment).

Une première partie de cette étude porte sur la détermination des courbes Proctor du sédiment avant et après traitement. Ces essais ont été effectués selon la norme NF P 98-231-1 en Proctor normal avec mesure de la portance. Pour le sédiment non traité, la teneur en eau a été réduite par séchage dans une étuve à une température de $50^{\circ} \mathrm{C}$ permettant une accélération du séchage sans modifier la nature du sédiment par dégradation des matières organiques. Pour le sédiment traité, le matériau est conservé, après malaxage, dans des bacs à l'air libre à une température entre 20 et $25^{\circ} \mathrm{C}$ pendant une période de 3 jours. A cet âge, le mélange est tamisé à travers un tamis d'ouverture de $5 \mathrm{~mm}$ pour simuler une opération de malaxage sur le terrain et accélérer le séchage naturel. Le mélange est ensuite conservé dans les mêmes conditions jusqu'à 7 jours. A cet âge, la teneur en eau du sédiment traité est encore assez élevée et atteint $80 \%$. Après un séchage à $50^{\circ} \mathrm{C}$, l'essai Proctor normal avec mesure de portance a été effectué. Dans la deuxième partie de cette étude, le matériau traité et conservé dans un container plastique fermé, a fait l'objet d'une étude de compressibilité suivant la norme XP P 94-090-1 pour des durées de conservation variables entre 3 et 58 jours. Après chaque essai de compressibilité, les échantillons sont observés par microscopie électronique à balayage (MEB) et analysés par diffractométrie aux rayons X (DRX) afin de suivre l'évolution de la microstructure et notamment le processus d'hydratation.

\section{RESULTATS ET DISCUSSION}

\subsection{COMPACTAGE}

D'après les courbes Proctor présentées dans la Figure 1, le sédiment non traité présente une masse volumique sèche maximale de $1,30 \mathrm{~g} / \mathrm{cm}^{3}$ à une teneur en eau optimale située à environ $30 \%$. Après traitement, on note un déplacement de l'optimum Proctor vers une masse volumique sèche maximale plus faible $\left(1,11 \mathrm{~g} / \mathrm{cm}^{3}\right)$ et une teneur en eau optimale de l'ordre de $38 \%$. Cet optimum Proctor ainsi que l'allure plate de la courbe semble être en accord avec les résultats de Dermatas (2003) qui 
montrent, pour des sédiments à des différentes teneurs en eau initiales $(90,130$ et $170 \%)$ et des dosages en ciment différents $(9,11$ et $13 \%)$, une teneur en eau optimale aux alentours de $40 \%$ et une masse volumique sèche maximale entre 0,90 et $1,2 \mathrm{~g} / \mathrm{cm}^{3}$. Concernant l'allure plate de la courbe de compactage, d'une part, elle serait liée à des limites de liquidités élevées (supérieures à $70 \%$ ) et aux fortes teneurs en matières organiques. D'autre part et d'un point de vue réalisation du compactage sur chantier, cet allure autorise une large gamme de teneurs en eau permettant d'atteindre $90 \%$ de la masse volumique sèche maximale.

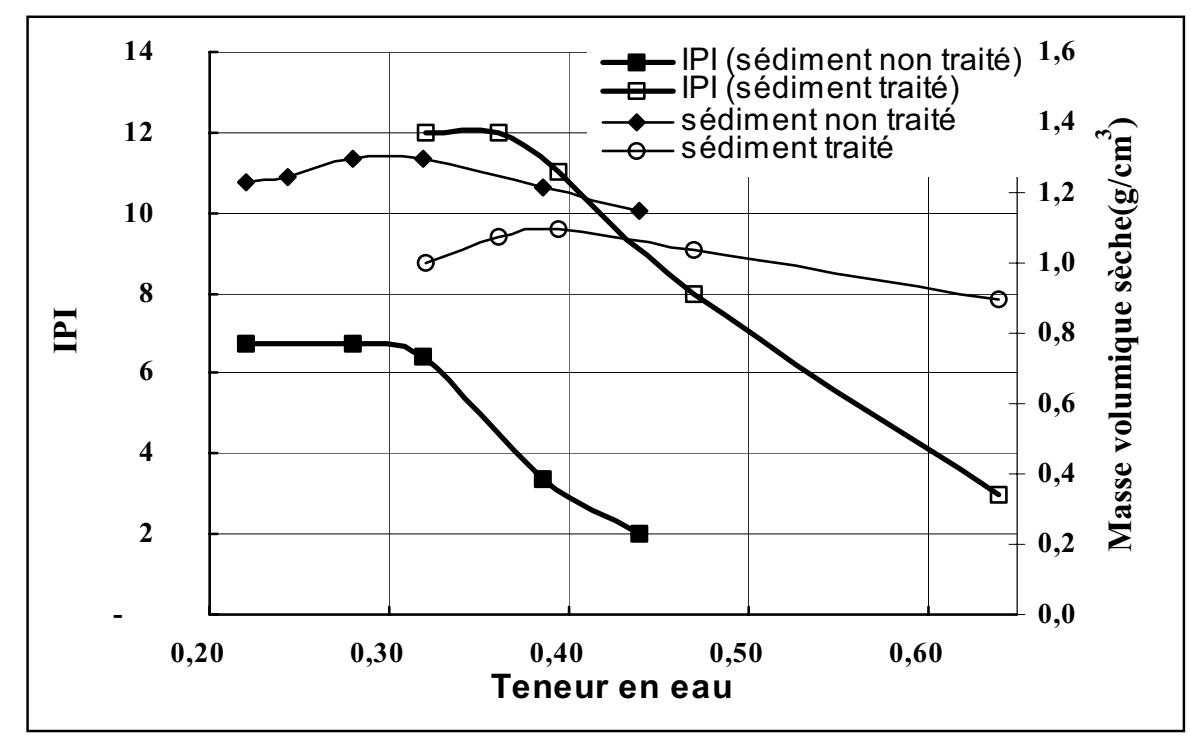

Figure 1: Courbe Proctor du sédiment avant et après traitement.

En ce qui concerne la portance du sédiment avant et après traitement, la Figure 1 montre la variation de l'indice portant immédiat en fonction de l'état hydrique du sédiment avant et après traitement. On y observe que le traitement a permis une amélioration de la portance puisque l'IPI, correspondant aux optimums, passe de 7 à 11 .

Les valeurs de l'indices portants immédiats obtenues démontrent que la valorisation des sédiments de dragage dans la réalisation des travaux type remblais est possible, notamment en France avec les sols de classe $A_{3}$ qui nécessitent des indices portant immédiat supérieur à 3 (GTS, 2000). De façon générale, la résistance mécanique peut être améliorée par ajout d'un correcteur granulométrique comme des sédiments plus grossiers par exemple.

\subsection{ETUDE DE LA COMPRESSIBILITE}

La compressibilité du sédiment a été étudiée avant et après traitement. Les courbes de compressibilité sont représentées sur la Figure 2. En ce qui concerne le sédiment brut, on observe que celui-ci ne montre aucune contrainte de préconsolidation. Cela signifie que la contrainte de préconsolidation est plus faible que la contrainte initiale appliquée qui était d'environ $15 \mathrm{kPa}$ ou qu'elle a été tout simplement effacée par le fait du remaniement. La compressibilité du sédiment parait importante même sous des faibles contraintes. Cela peut être attribué à la quantité importante de matières organiques $(9 \%)$ qui ont tendance à former des complexes argilo-organiques, augmentant ainsi l'indice des vides et par conséquent la compressibilité (Stevenson, 1994, dans Tremblay et al. 2001). Après traitement, des pressions de préconsolidation (Figure 2 et Tableau 2) se sont développées dans le matériau et ont tendance à augmenter pendant les premiers jours pour atteindre $95 \mathrm{kPa}$ à 3 
jours, puis $300 \mathrm{kPa}$ à 14 jours. Elles diminuent ensuite progressivement jusqu'à $240 \mathrm{kPa}$ à 58 jours. L'apparition des pressions de préconsolidation est très probablement due à l'effet de cimentation résultant de l'ajout du liant hydraulique à savoir le ciment. Les analyses de la microstructure présentée ci-après confirment la modification de la structure du matériau traité par développement des hydrates du ciment. Si on s'intéresse à la variation de l'indice de compressibilité $\mathrm{C}_{\mathrm{c}}$ du sédiment traité en fonction du temps on note qu'il est de $0,41 \pm 0,03$ pour les différents âges.

\begin{tabular}{|c|c|c|c|}
\hline Echantillon & Age (jour) & $\begin{array}{c}\text { Pression de préconsolidation } \\
(\mathrm{kPa})\end{array}$ & Indice de compression $\mathrm{C}_{\mathrm{c}}$ \\
\hline Sédiment non traité & - & - & 0,29 \\
\hline S3J10C & 3 & 95 & 0,41 \\
\hline S7J10C & 7 & 286 & 0,39 \\
\hline S14J10C & 14 & 300 & 0,41 \\
\hline S21J10C & 21 & 228 & 0,38 \\
\hline S28J10C & 28 & 259 & 0,44 \\
\hline S58J10C & 58 & 240 & 0,40 \\
\hline
\end{tabular}

Tableau 2. Pressions de préconsolidation et indices de compression du sédiment avant traitement et pour différents âges après traitement.

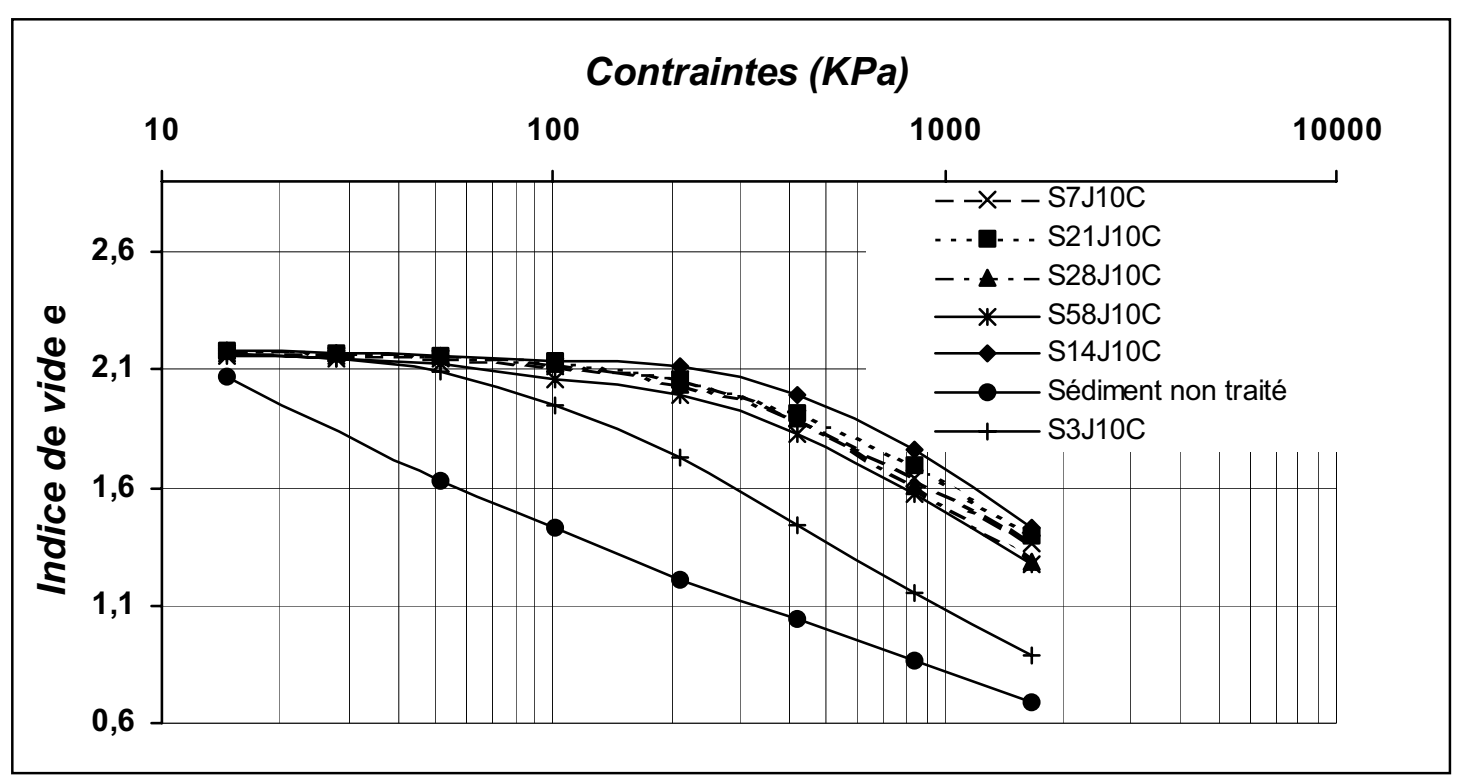

Figure 2: Courbes de compressibilité du sédiment avant traitement et pour différents âges après traitement.

Lors des essais de consolidation il n'a pas été possible d'atteindre la fin de consolidation du sédiment non traité et sous des faibles contraintes après traitement. De ce fait, la détermination du temps de fin de consolidation, des coefficients de consolidation et des indices de consolidation secondaire n'a pu être effectuée que pour des incréments de charge induisant des pressions proches ou supérieures à la pression de préconsolidation. Dans ce cas on note que le comportement en compressibilité du sédiment traité est fortement modifié. Le Tableau 3 donne les rapports entre les 
différents paramètres de consolidation sous la plus forte contrainte appliquée (environ $1670 \mathrm{kPa}$ ). Ces résultats ont été obtenus selon la méthode de Casagrande.

\begin{tabular}{|c|c|c|c|}
\hline Echantillon & $\begin{array}{c}\text { Coefficient } \mathrm{C}_{\mathrm{v}} \\
\left(\mathrm{m}^{2} / \mathrm{an}\right)\end{array}$ & $\mathrm{t}_{\mathrm{p}}(\mathrm{AT}) / \mathrm{t}_{\mathrm{p}}(\mathrm{NT})$ & $\mathrm{C}_{\alpha}(\mathrm{AT}) / \mathrm{C}_{\alpha}(\mathrm{NT})$ \\
\hline $\begin{array}{c}\text { Sédiment non } \\
\text { traité }\end{array}$ & 1,26 & 1 & 1 \\
\hline S3J10C & 1,69 & 0,4 & 0,62 \\
\hline S58J10C & 8,46 & 0,4 & 0,44 \\
\hline
\end{tabular}

NT : Paramètre correspond au sédiment non traité, AT : Paramètre correspond au sédiment après traitement.

Tableau 3 : Effets du traitement sur la compressibilité du sédiment avant et après traitement.

Pour le sédiment non traité, le temps de consolidation est important et est caractéristique des sols fins organiques. Ainsi, la fin de la consolidation on n'a pu être atteinte qu'après trois jours et sous la plus grande contrainte appliquée (environ $1670 \mathrm{kPa}$ ). Après traitement, le temps de consolidation $t_{p}$, est largement réduit pour des contraintes proches ou supérieures à la pression de préconsolidation. A titre d'exemple et à l'âge de trois jours, $\mathrm{t}_{\mathrm{p}}$ a été réduit de plus de la moitié. Quant au coefficient de consolidation primaire $\mathrm{C}_{\mathrm{v}}$, il s'est avéré faible pour le sédiment brut (environ 1,26 $\mathrm{m}^{2} / \mathrm{an}$ ), reflétant un processus de consolidation primaire lent et une faible perméabilité. 58 jours après traitement, ce coefficient augmente et atteint $8,46 \mathrm{~m}^{2} /$ an ( 7 fois plus grand que celui du sédiment non traité).

Le tassement secondaire est un aspect important dans l'étude de la consolidation des sédiments et sols fins. Celui-ci est à l'origine de désordres rencontrés dans les constructions type remblais (tassement différentiel). Après traitement au ciment, le coefficient de consolidation secondaire $\mathrm{C}_{\alpha}$ est réduit à près de $2 / 3$ de sa valeur initiale $\left(\mathrm{C}_{\alpha}=0,0078\right.$ pour le sédiment non traité et une contrainte de $1670 \mathrm{kPa})$ et à moins de la moitié après 58 jours $\left(\mathrm{C}_{\alpha}=0,0034\right)$.

Pour un sol donné, le rapport $\mathrm{C}_{\alpha} / \mathrm{C}_{\mathrm{c}}$ est constant. Dans le cas de notre sédiment non traité il est de 0,027. Après traitement, on constate une diminution de ce rapport au fur et à mesure de la période de cure du sédiment traité. Il est de 0,012 après 3 jours et de 0,0086 après 58 jours. Pour des sols inorganiques, le rapport $\mathrm{C}_{\alpha} / \mathrm{C}_{\mathrm{c}}$ diminue avec l'augmentation des tailles des grains du sol (Tao-Wei Feng et al. 2001). Ceci met en évidence l'effet de cimentation et de floculation qu'a subi la structure du sédiment après traitement au ciment.

\subsection{MICROSTRUCTURE}

La Figure 3 montre certains aspects de la microstructure du sédiment avant et après traitement. La micrographie (a) montre la nature des constituants du sédiment avant traitement et où une quantité importante de matières organiques est présente. Suite à l'incorporation du ciment, on constate une évolution de la microstructure du sédiment traité avec la formation de composés hydratés dont les plus visibles sont l'ettringite et la portlandite (micrographie (b) de la Figure 3). L'analyse par DRX du sédiment brut et traité a été effectuée afin de mieux cerner les changements de la minéralogie engendrés par l'ajout de ciment. En ce qui concerne le sédiment brut, les analyses ont montré que les principaux minéraux présents sont le quartz et la calcite. D'autres minéraux, tels que la pyrite, l'halite et le feldspaths ont été détectés. La fraction argileuse semble être constituée de smectite, d'illite, de mica et de kaolinite. L'analyse de plusieurs spectres de diffraction correspondant à différentes périodes 
de cure des échantillons du sédiment traité a montré une évolution de la minéralogie de la structure. L'exemple du sédiment traité et âgé de 58 jours est présenté à la Figure 4 et indique qu'en plus des minéraux propres au sédiment brut, plusieurs composés hydratés, types C-S-H, C-A-S-H, ettringite et portlandite, se sont développés dans la nouvelle matrice du sédiment traité. Cette nouvelle microstructure semble avoir une influence sur la compressibilité et ce dès les premiers jours après traitement. Ainsi, l'apparition de composés semble augmenter la pression de préconsolidation et réduit la compressibilité des sédiments traités, notamment sous des contraintes inférieures à la pression de préconsolidation. L'étude approfondie de la microstructure se poursuit actuellement afin de mieux cerner son rôle vis-à-vis du comportement géotechnique.

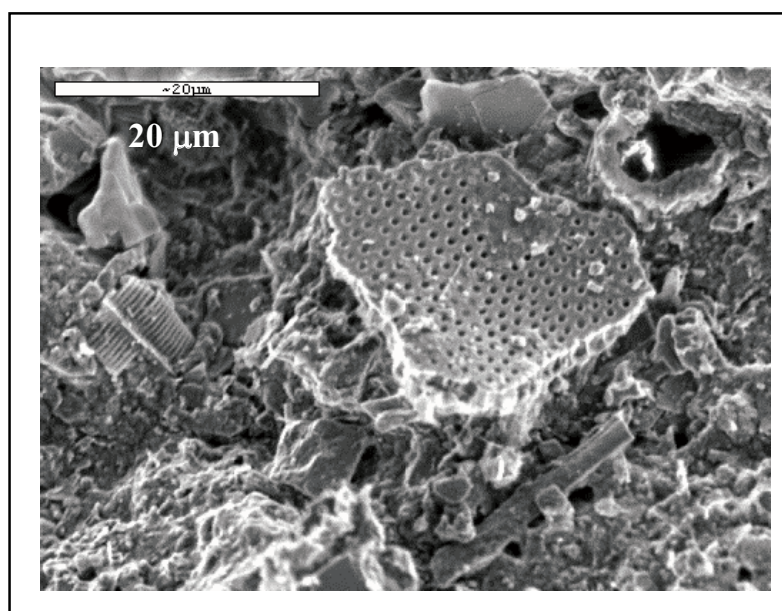

(a) Sédiment avant traitement.

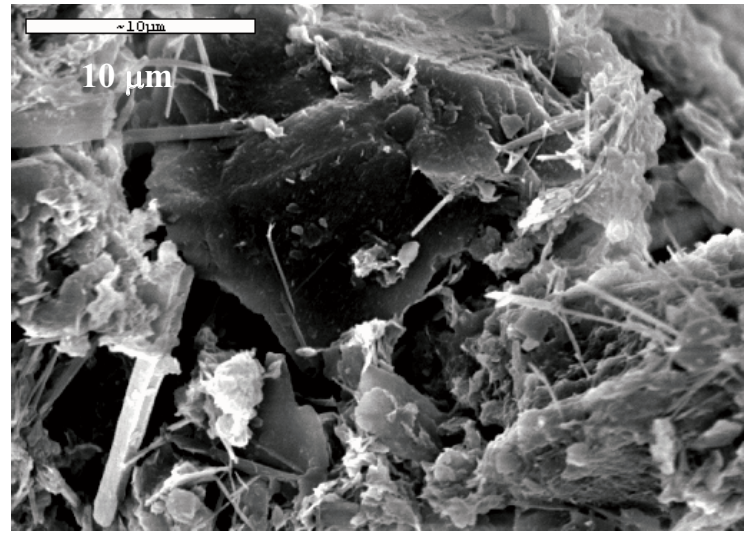

c) Sédiment traité et après 7 jours de cure

Figure 3 : Micrographies MEB de la microstructure du sédiment avant et après traitement au ciment.

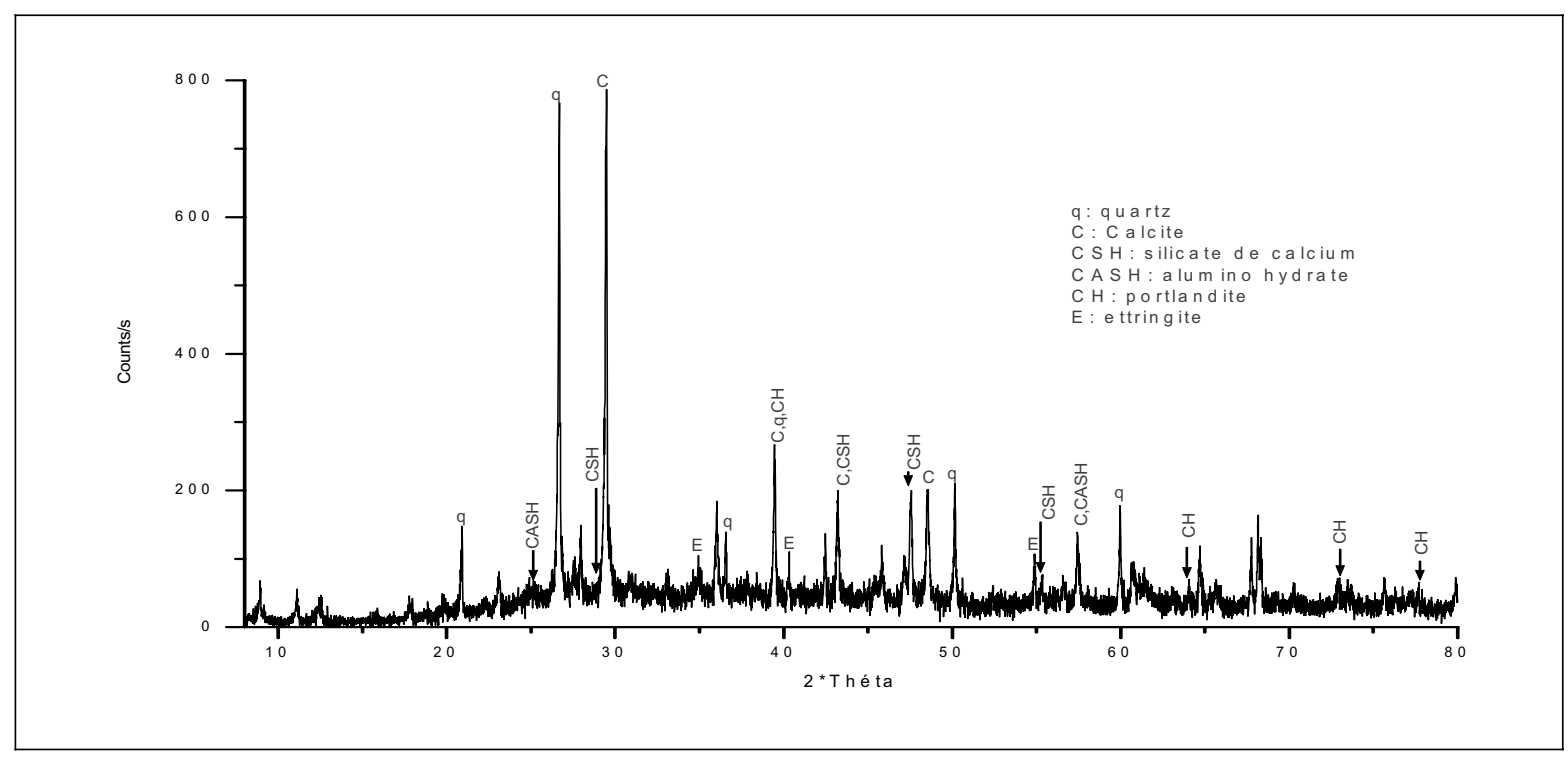

Figure 4 : Spectre de diffraction du sédiment après traitement (58 jours de cure). 


\section{CONCLUSION}

D'après les premières investigations sur les propriétés géotechniques d'un sédiments de dragage traitée avec $10 \%$ ciment, les conclusions suivantes peuvent être énoncées :

$>$ Le sédiment traité au liant hydraulique présente une possibilité d'utilisation comme matériau de remblaiement. Par rapport au sédiment brut, le sédiment traité présente une portance améliorée et une teneur en eau optimale élevée. Vu les teneurs en eau initiales élevées des sédiments bruts, une solution alternative à une déshydratation coûteuse pourrait être l'incorporation d'autres ajouts consommateurs d'eau et d'éléments grossiers.

$>$ La compressibilité du matériau traité est largement réduite et son comportement devient plus proche des sols grenus que de ceux des sols fins sous l'effet de cimentation et de diminution de l'effet de la matière organique. L'effet de l'ajout du ciment a également eu pour conséquence l'apparition d'une pression de préconsolidation dès les premiers jours de cure. La pression de préconsolidation atteint environ $250 \mathrm{kPa}$.

Les analyses de la microstructure par DRX et MEB confirment le développement par cimentation de composés hydratés qui ont permis l'apparition d'une pression de préconsolidation et la compressibilité de façon générale.

Remerciements : Les auteurs tiennent à remercier MM. F. Gourbilleau et C. Dufour du laboratoire du SIFCOM de l'ENSICAEN pour leur aide et leur collaboration.

\section{REFERENCE}

Bennert T.A, Maher M.H, Jafari F, Gucunski N.(2000 « Use of dredged sediments from Newark Harbor for geotechnical applications ». Geotechnics of high water content materials, ASTM STP 1374, T. B. Edil and P. J. Fox, Eds., ASTM, West Conshohocken, p. 153-164.

Boutouil. (1998) «Traitement des vases de dragage par solidification/stabilisation à base de ciment et additifs ». Thèse de doctorat, Université du Havre.

Colin. D. (2003) «Valorisation des sédiments fins de dragage en technique routière». Thèse de doctorat, Université de Caen.

Dermatas D, Dutko P, Balorda-Barone J, Moon D. (2003) «Evaluation of engineering properties of cement treated Hudson River dredged sediments for reuse as fill material ». Journal of marine environmental engineering, 7, p. 101-123.

GTS (2000) « Traitement des sols à la chaux et aux liants hydrauliques -Guide technique ». Edition LCPC- SETRA.

Keller G.H., (1982) « Organic matter and the geotechnical properties of submarine sediments ». Geomarine letters, vol. 2 , p. 191-198.

Rashid et Brown (1975) «Influence of marine organic compounds on the engineering properties of remoulded sediment ». Engineering geology, 9, p. 141-154.

Tao-Wei F, Jia-Yih L, Yi-Jiuan L. (2001) « Consolidation behaviour of soft mud treated with small cement content » Engineering geology, 59, p. 327-335.

Tremblay H, Leroueil S, Locat J. (2001) « Mechanical improvement and vertical yield stress prediction of clayey soils from eastern Canada treated with lime and cement ». Canadian geotechnical journal. 38: 567-579. 